\title{
UNIVERSIDAD, INTERCULTURALIDAD Y DISCURSO PÚBLICO. LA UNIVERSIDAD EN UN CONTEXTO SOCIAL DEMOCRÁTICO.
}

Resumen. La universidad constituye el lugar privilegiado de la sociedad civil en el que los ciudadanos buscan nuevos conocimientos y se preparan para participar como expertos en la tematización, discusión y resolución de todas aquellas cuestiones teóricas y prácticas relevantes para la sociedad. La educación universitaria tiene que contribuir, por consiguiente, a la formación de docentes e investigadores, tecnólogos y expertos en las diversas esferas del saber, y a la capacitación para la reflexión crítica a través del desarrollo de competencias discursivas, éticas y culturales. En este sentido, los universitarios son -tendrían que ser- personas capacitadas no sólo para aumentar metódica y sistemáticamente el conocimiento, sino también para reflexionar críticamente sobre cuestiones centrales de la convivencia humana en un sistema democrático. Por ello, los universitarios tendrían que ser profesionales y expertos, a la vez que "personas de principios", conocedoras de su propia cultura y del contexto histórico de aplicación.

Palabras claves: Universidad - interculturalidad - ética - ciudadanía - democracia.

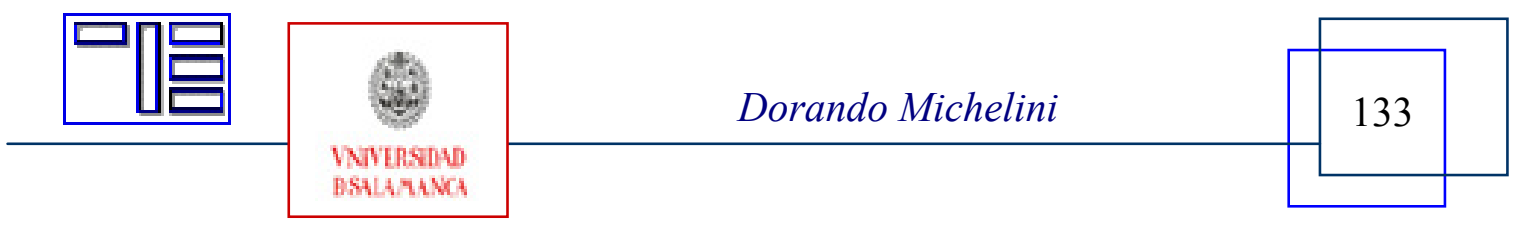




\section{UNIVERSITY, INTERCULTURALITY AND PUBLIC DISCOURSE. THE UNIVERSITY IN A SOCIAL-DEMOCRATIC CONTEXT.}

Abstract. The university constitutes the privileged place of civil society in which citizens seek new knowledge and prepare themselves to participate as experts in the discussion and resolution of all theoretical and practical issues relevant to society. University education has to contribute, therefore, to the formation of teachers and researchers, technologists and experts in the diverse spheres of knowledge, and to the training for critical thinking through the development of ethical, cultural and discursive skills. In this regard, university graduates are - should be- people qualified not only to enlarge their knowledge methodically and systematically, but also to reflect critically on central questions of human coexistence in a democratic system. Thus, university graduates would have to be professionals and experts as well as "people of principles", proficient in their own culture and in the historical context of implementation.

Key Words: University - interculturality - ethics - citizenship - democracy.

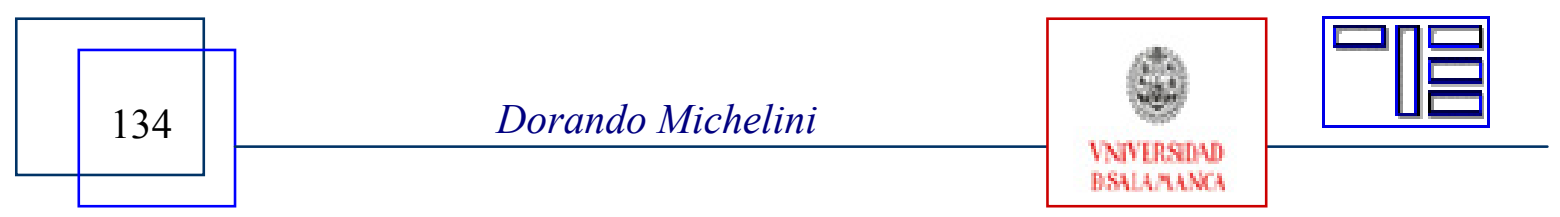




\section{UNIVERSITÉ, INTERCULTURALITÉ ET DISCOURS PUBLIC. L'UNIVERSITE DANS UN CONTEXTE SOCIAL-DÉMOCRATIQUE.}

Sommaire. L'Université constitue l'endroit privilégié de la société civile où les citoyens cherchent de nouvelles connaissances et se préparent pour participer en tant qu'experts, à la thématisation, la discussion et la résolution de toutes les questions théoriques et pratiques remarquables pour la société. En conséquence, l'éducation universitaire doit contribuer à la formation d'enseignants et de chercheurs, de technologues et d'experts dans les diverses sphères du savoir et à la préparation à la réflexion critique à travers le développement de compétences discursives, éthiques et culturelles. Dans ce sens, les universitaires sont -devraient être- des personnes prêtes, non seulement à accroître méthodiquement et systématiquement la connaissance mais aussi à réfléchir de manière critique sur les questions centrales de la vie en commun dans un système démocratique. Les universitaires devraient être des professionnels et des experts, mais en même temps des «gens de principes», connaisseurs de leur propre culture et du contexte historique d'application.

Mots- clés: Université - interculturalité - éthique - citoyenneté - démocratie.

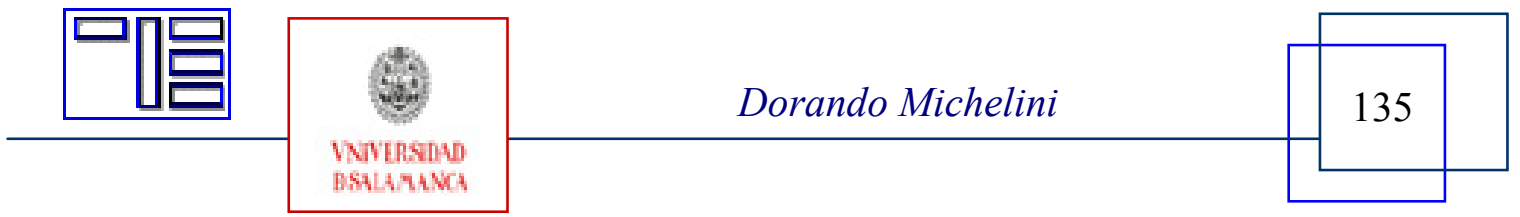


Revista Electrónica Teoría de la Educación.

Educación y Cultura en la Sociedad de la Información.

http://www.usal.es/teoriaeducacion

Vol. 9. No 2. Mayo 2008

UNIVERSIDAD, INTERCULTURALIDAD Y DISCURSO PÚBLICO. LA UNIVERSIDAD EN UN CONTEXTO SOCIAL DEMOCRÁTICO.

\author{
Dorando J. Michelini \\ dmichelini@arnet.com.ar \\ CONICET, Agencia Córdoba Ciencia - Universidad Nacional de Río Cuarto \\ y Fundación ICALA (Argentina).
}

\title{
1.- DESARROLLO CIENTÍFICO-TECNOLÓGICO E INTERCULTURALIDAD.
}

La crisis económica, social, política, ecológica y cultural del mundo contemporáneo tiene que ver, en gran medida, con los avances de la ciencia y las consecuencias del desarrollo científico-tecnológico, pero también con la dificultad de visualizar una solución razonable para la cuestión de la diversidad cultural y, con ello, de hallar parámetros y normas intersubjetivamente válidos de entendimiento que sirvan de orientación para las decisiones y acciones colectivas de la humanidad en un mundo globalizado e intercultural.

La Universidad constituye el lugar privilegiado de la sociedad civil en el que los ciudadanos buscan nuevos conocimientos y se preparan para participar como expertos en la tematización, discusión y resolución de todas aquellas cuestiones teóricas y prácticas relevantes para la sociedad. La educación universitaria tiene que contribuir, por consiguiente, a la formación de docentes e investigadores, tecnólogos y expertos en las diversas esferas del saber, y a la capacitación para la reflexión crítica a través del desarrollo de competencias discursivas, éticas y culturales. En este sentido, los universitarios son -tendrían que ser- personas capacitadas no sólo para aumentar metódica y sistemáticamente el conocimiento, sino también para reflexionar críticamente sobre cuestiones centrales de la convivencia humana en un sistema democrático. Por ello, los universitarios tendrían que ser profesionales y expertos, a la vez que "personas de principios", conocedoras de su propia cultura y del contexto histórico de aplicación.

\subsection{La ética y los desafíos científico-tecnológicos.}

Muchos de los problemas éticos contemporáneos están intrínsecamente relacionados con el proceso de globalización y sus consecuencias económicas, políticas y culturales. La globalización no es un proceso unívoco: ella puede ser interpretada como un fenómeno irreversible de interacción a nivel planetario, pero también como mito o ideología. (Bourdieu, 1999). Dado que la globalización produce no sólo más y mejores posibilidades de comunicación, de conocimientos y de intercambio, sino también mayor

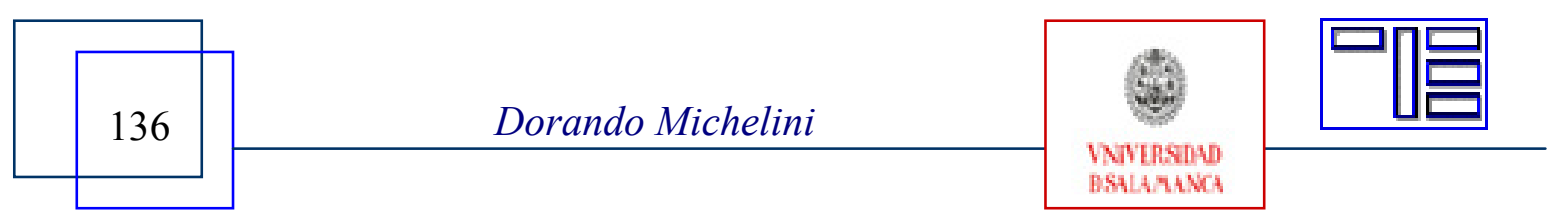


desigualdad y exclusión, la reflexión crítica es imprescindible para discernir entre los verdaderos aportes de la globalización y sus graves consecuencias a nivel social, ético y cultural (consecuencias que se perciben de un modo peculiar, por ejemplo, en las posibilidades de ingreso a la universidad y en las desiguales oportunidades de acceso al conocimiento, que terminan siendo también desigualadoras formas de ejercicio de la ciudadanía).

Desde una perspectiva ético-filosófica, no son pocos los autores que han señalado que el desarrollo de la ciencia plantea a la humanidad no sólo problemas técnicos, económicos, sociales y políticos, sino también cuestiones éticas y culturales inéditas (Jonas, 1985, 1995; Apel, 1973, 1988) Así, por ejemplo, en la primera mitad del siglo XIX, las ciencias físicas provocaron no sólo una revolución en las comunicaciones, las computadoras y los medios de transporte, sino también en la producción de armas nucleares (Nossal, 1988), con las cuales el poder alcanzado por el ser humano comenzó a ser un problema para la propia subsistencia de la humanidad como especie. En la segunda parte del siglo XX, con los avances de la ingeniería genética y la posibilidad de la clonación humana, el poder humano se amplía y profundiza. A partir de la década de 1950, el estudio de la estructura y la función del ADN se convierte en un tema central de la biología. "En menos de una década, se hizo evidente que la ingeniería genética y las tecnologías afines representaban el mayor avance de las ciencias de la vida en este siglo" (Nossal, 1988: 12). Claro está que, junto al deslumbramiento que causan los resultados que pueden alcanzarse a través de la ingeniería genética en el ámbito terapéutico humano para la mejor comprensión y la eliminación de enfermedades; en el económico, por las generosas recompensas a la industria, y en el agrícolo-ganadero, por los éxitos de la manipulación genética en los rendimientos de la producción; en el control de enfermedades, en el aceleramiento del crecimiento en la producción pecuaria; etcétera, aparecen también no sólo el temor por las consecuencias de una expansión sin límites de la experimentación y la manipulación de la vida, sino también reservas en relación con la licitud moral y ética en orden a resguardar la dignidad de las personas y a lograr un desarrollo humano justo y emancipatorio. Aquí reaparece, bajo nuevos y fascinantes aspectos, la antigua cuestión ética de la relación entre el poder-hacer y del deber-hacer.

Los debates sobre la necesidad y posibilidad de dar una respuesta ética a estos problemas tienen, en efecto, una larga tradición. Mientras algunos siguen afirmando que la ciencia, en general, "es sin punto de comparación la empresa de mayor éxito que hayan acometido nunca los seres humanos" (Nossal, 1988: 152) y -de acuerdo a la afirmación de Marcelin Barthelot (cit. por Nossal, 1988: 152)- "brindará la base verdaderamente humana de la moral y la política en el futuro", otros sostienen que el desarrollo científico-tecnológico acarrea no sólo consecuencias deshumanizantes, que anuncian el final de un tipo civilizatorio, sino que atentan directamente contra la supervivencia de la especie humana y, en general, contra la vida en el planeta, y exigen un giro radical en el modo de pensar y actuar de la humanidad en su conjunto. (Jonas, 1995). Si se percibe la ambigüedad de la técnica, es posible coincidir en que ya no es

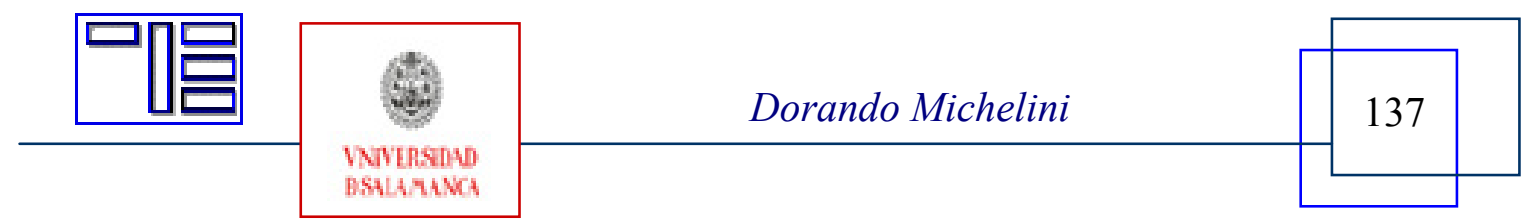


Revista Electrónica Teoría de la Educación.

Educación y Cultura en la Sociedad de la Información.

http://www.usal.es/teoriaeducacion

Vol. 9. No 2. Mayo 2008

posible sostener que "el hombre técnico tiene que aplicar todo lo que comprende" y, en ello, "no fijarse límites" (Edward Teller, seg. Lenk y Ropohl, 1987: 6).

En el siglo XX, numerosos filósofos han tratado explícitamente el problema de las dramáticas consecuencias del desarrollo científico tecnológico, ${ }^{1}$ pero recién en la década de los años setenta, con obras como las de Hans Jonas (1985, 1995), se comienza a percibir en toda su profundidad la problemática de la intervención técnica del ser humano en el mundo natural y sus consecuencias planetarias. Desde entonces, las discusiones sobre la tecnología y su impacto en el medio ambiente y en la sociedad, particularmente con los avances de la ingeniería genética, se expanden rápidamente. (Böhme, 1992; Irrgang, 2002).

En la actualidad, los desafíos éticos que plantean el desarrollo científico-tecnológico y sus consecuencias globales vuelven a tener así una relevancia inusitada. La clásica articulación ética entre "poder" y "deber" -no todo lo que se puede hacer se debe hacervuelve a plantearse en diversos ámbitos: sin dejar de problematizar aquellos aspectos que se pueden y deben hacer para alcanzar una "vida buena", la reflexión ética se ha concentrado en la problemática de la vida en general, y de la vida humana en particular (en lo que se puede y se debe hacer en relación con la supervivencia de la especie; en relación con el comienzo y final de la vida humana, etc.).

En vista de que los seres humanos han aprendido a hacer todo lo que pueden, pero no han aprendido a limitar libremente su poder -esto es, a hacer lo que deben-, hay dos cuestiones éticas clave para pensar: por un lado, una fundamentación adecuada y suficiente de la responsabilidad humana en relación la competencia técnico-científica, y, por otro lado, la cuestión de una aplicación informada, reflexionada y eficaz de una ética competente para el mundo científico-tecnológico y sus consecuencias sociales, económicas, políticas y culturales.

\subsection{La ética y los desafíos de la interculturalidad.}

Paralelamente a estos debates, se discute también los desafíos a los que se enfrenta la ética en vista de la fundamentación de normas en contextos de acción interculturales y conflictivos. "La interculturalidad es una nueva toma de conciencia cada vez más nítida de que todas las culturas están en un proceso de gestación de sus propios universos de sentido, y que no existe la posibilidad teórica de subsumir completamente al otro en mi sistema de interpretación" (Salas Astrain, 2003: 79). La interculturalidad se refiere tanto a la diversidad de culturas emergentes como a los respectivos reposicionamientos de las culturas entre sí en un mundo común. Estos reposicionamientos -es decir, estos encuentros y desencuentros entre culturas- están signados no sólo por la búsqueda de comprensión y entendimiento con los otros, sino también por el conflicto, el desentendimiento y la violencia.

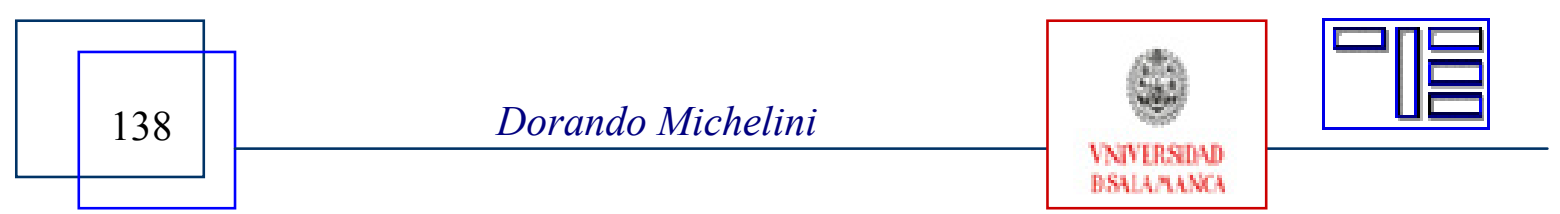


Una de las características de las modernas sociedades democráticas -abiertas y pluralistas- en la era de la globalización es la amplitud y heterogeneidad de teorías e ideologías que compiten en el ofrecimiento de sentido y de puntos de vista diversos y divergentes en lo que se refiere a la legitimación y crítica de los modelos de pensamiento y acción. Hasta la fecha, y por muy diversas razones teóricas y prácticas, ninguno de los distintos modelos contemporáneos científicos, políticos, ideológicos, filosóficos y teológico-religiosos ha logrado imponerse como parámetro intersubjetivamente válido de legitimación y crítica, ni sus respectivas pretensiones de validez teórica y de vincularidad práctica han podido alcanzar el asentimiento libre e irrestricto de todos los afectados. (Michelini, 1998, 2000).

La con-vivencia aparece así como una fenómeno clave de la filosofía intercultural, signado por la conflictividad, cuya construcción requiere de un diálogo amplio y eficaz en orden a la configuración de lo humano: se trata de "un complejo diálogo entre eticidades que nos debe poner en condiciones de reencontrarnos con la "medida" de la convivencia solidaria como "medida" de todas las "medidas" que nos trasmiten nuestras memorias culturales contextuales" (Fornet Betancourt, 2003: 10). En este sentido, pensar la con-vivencia como un resultado de la libertad, del reconocimiento del otro y del acuerdo mutuo implica un serio desafío para toda reflexión ético-filosófica, tanto en orden a fundamentar normas intersubjetivamente válidas, como también en lo que respecta a una aplicación razonable, eficaz y compartida en contextos históricos y culturales diversos. En la actualidad, este diálogo puede ser visualizado y concretado como un discurso público, abierto y pluralista, de todos los implicados y afectados.

\section{2.- UNIVERSIDAD Y CIENCIA.}

Desde un punto de vista histórico, en los inicios de la modernidad comienza a cuestionarse la idea de universidad en la que la filosofía tenía la pretensión de constituirse, en cuanto ciencia pura, en la suministradora de unidad de las ciencias. Este cuestionamiento no surge, sin embargo, del interior de la universidad sino de la nueva ciencia (de la ciencia matemática y experimental de la naturaleza) que comienza a gestarse en las academias y centros de investigación independientes de la universidad. (Apel, 1987) El perfil humanístico de la universidad -como lugar de búsqueda universal de la verdad, de la teoría en sentido griego y de la antigua concepción de la retóricacomienza a ser suplantado así, a partir del siglo XVII, por una concepción de la universidad como prestadora de servicios, que se va adaptando progresivamente a las exigencias sistémicas epocales del desarrollo de la ciencia de la naturaleza (Apel, 1987) $\mathrm{y}$, con ello, a los intereses utilitaristas y a la exigencia de producir resultados cuantificables. En la actualidad, esta tendencia a la cuantificación y al utilitarismo ha alcanzado incluso un alto grado de potenciación en el fenómeno de la comercialización del saber. ${ }^{2}$ Las exigencias de la teoría y del saber puro filosófico van siendo sustituidas por los requerimientos del mercado: por la demanda de saber técnico y de aplicación económica utilitaria, y por la formación de expertos, especialistas y profesionales. En

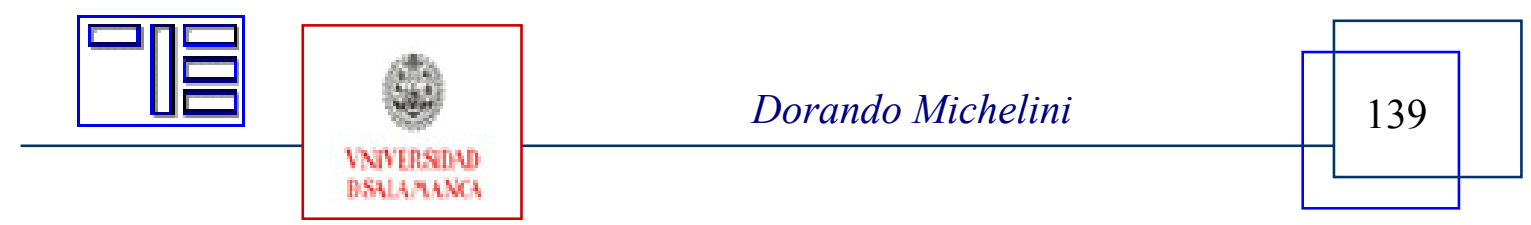


Revista Electrónica Teoría de la Educación.

Educación y Cultura en la Sociedad de la Información.

http://www.usal.es/teoriaeducacion

Vol. 9. No 2. Mayo 2008

los siglos XIX y XX, el a priori técnico de las ciencias naturales se devela y consolida finalmente como "la primera fuerza productiva de la sociedad industrial moderna" (Apel, 1987). También los docentes, los estudiantes y la administración universitaria se van adaptando a las exigencias de la eficacia y productividad.

En los siglos XIX y XX, con el surgimiento del positivismo y el auge del neopositivismos, se alcanza una nueva unificación de los intereses y la metodología científica, pero a costa de una insostenible reducción de los intereses y los métodos de las Ciencias Humanas a la metodología de las ciencias empírico-analíticas y naturales. Este intento de unificación fracasa porque la base sintáctico-semántica de sustentación conceptual y teórica, y la perspectiva metodológica de la neutralidad valorativa resultan demasiado abstractas y estrechas para explicar la diversidad de los intereses que orientan el conocimiento y la multiplicidad de los fines sociales y culturales. Con ello, la ciencia no es capaz ya de suministrar parámetros normativos para la legitimación y crítica de las formas de vida.

En la segunda mitad del siglo XX, con el auge de la ingeniería genética y de la informática, y el avance de la globalización, se amplía y consolida, por un lado, la tendencia de institucionalizar la neutralidad valorativa en el todos los subsistemas de la sociedad: tanto en la economía y la política, como en el derecho y la educación. En este contexto, la reflexión filosófica, la moral y la religión, en cuanto sistemas de orientación y crítica de las acciones y decisiones humanas, se debilitan y pierden incidencia en el ámbito público. Más aún, la filosofía, la moral y la religión terminan, demasiado a menudo, adaptándose funcionalmente al sistema.

Por otro lado, las críticas radicales a la racionalidad, que hunden sus raíces en aquellas comprensiones filosóficas que articulan el logos humano exclusiva o fundamentalmente con la contingencia y el poder, no han podido tampoco remediar esta situación. Las discusiones teórico-metodológicas -iniciadas por Dilthey en relación con las ciencias del espíritu y profundizadas y ampliadas por Heidegger con sus planteos sobre la historia del ser y la problemática de la técnica- se prolongan hasta nuestros días y alcanzan un punto culminante en la disputa de métodos entre el reduccionismo orientado a las ciencias naturales y el historicismo hermeneuticista de las ciencias humanistas. (Adorno, 1987) Más aún, en la actualidad, propuestas provenientes del posmodernismo proclaman el fin de las ideologías (Lyotard, 1989) y pensadores como Rorty abogan directamente por un contextualismo radical y consecuente, y apuestan por el abandono de la filosofía. (Rorty, 1983).

Ante este panorama, surgen preguntas como las siguientes: La universidad: ¿debe seguir fomentando el saber científico en dirección a su aplicabilidad técnica y su utilización económica? ¿Cuál es el papel de la Universidad en orden a la investigación articulada con el desarrollo? ¿Sólo le cabe capacitar para producir expertos, especialistas, profesionales? ¿La universidad, debe abandonar definitivamente el saber especulativo? Si esto es así: ¿está condenada la filosofía a ser expulsada definitivamente de la

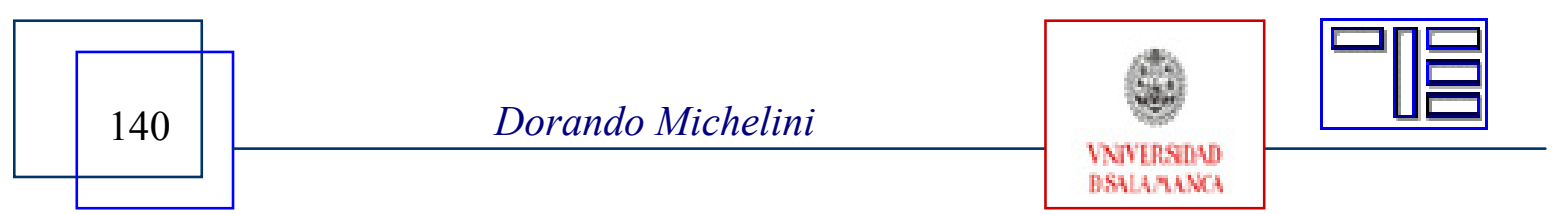


Universidad? Todo se sintetiza, finalmente, en esta cuestión: ¿es necesario y posible, en un mundo global e intercultural, alcanzar una articulación entre el saber científícotécnico y el saber filosófico?

\section{3.- UNIVERSIDAD Y DISCURSO PÚBLICO.}

La Universidad es -debe ser- el lugar donde se examina a fondo la realidad con los métodos investigativos propios de las distintas disciplina y, a la vez, donde se dilucidan conjunta y críticamente los tortuosos procesos humanos de ilustración a nivel teórico y práctico, que tienen como fin último acrecentar el conocimiento, aumentar el bienestar y crear posibilidades de autorrealización para el ser humano. La Universidad es -debe sertambién un lugar privilegiado del ejercicio de la corresponsabilidad solidaria en orden a la configuración de un mundo más humano: su capacidad de análisis y crítica vigilante frente a los nuevos conocimientos y sistemas de orientación tiene que convertirse en el fundamento de una sociedad democrática.

\subsection{Discurso científico-tecnológico y discurso crítico.}

La Universidad es un lugar privilegiado para la articulación del discurso científicotecnológico y el discurso crítico. En este sentido, la tarea de la Universidad consiste tanto en un esfuerzo por mejorar la formación científico-investigativa, orientada al aumento del saber, como así también a la capacitación profesional.

La articulación de ambos principios puede alcanzarse, por un lado, a nivel del saber, en la medida en que es posible la interdisciplinariedad y un diálogo permanente entre la forma de investigación, los métodos y los resultados de las ciencias particulares y la reflexión filosófica. Las ciencias están encaminadas a un constante aumento del conocimiento a través de investigaciones sistemáticas, pero la humanidad parece no disponer de normas éticas suficientemente fundadas y efectivas para orientar la interacción colectiva. Y esta realidad es tanto más preocupante, por cuanto en la actualidad ya no se trata de la disputa entre las Ciencias de la Naturaleza y las Ciencias Humanas, sino más bien del eclipsamiento de las Ciencias Humanas frente al auge de las Ciencias de la Vida (la biología, la genética, la biotecnología, etc.). Los desafíos teóricos y prácticos que se presentan en estas investigaciones sobrepasan largamente las capacidades actuales de respuesta teórica y práctica del ser humano, y muchas consecuencias directas e indirectas de la ciencia y de la tecnología están acarreando problemas dramáticos para la existencia misma de la humanidad en su conjunto.

Por otro lado, dado que la universidad es una institución que no sólo aporta a la competencia y competitividad en el ámbito profesional y laboral, sino que también tiene una función crítica y moral (Roig, 1998), la formación de los profesionales tiene que estar organizada de modo que los universitarios puedan recibir no sólo una capacitación excelente a nivel de conocimientos específicos y especializados, inherentes a las

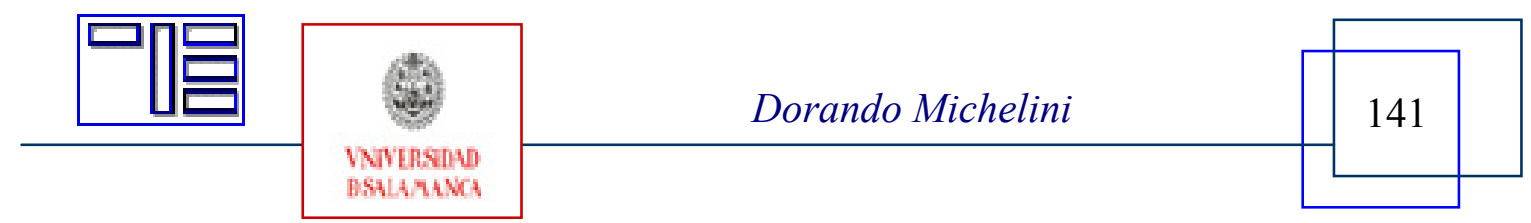


Revista Electrónica Teoría de la Educación.

Educación y Cultura en la Sociedad de la Información.

http://www.usal.es/teoriaeducacion

Vol. 9. No 2. Mayo 2008

distintas disciplinas, sino también las oportunidades y los elementos necesarios para ampliar y profundizar su competencia crítica en orden a su integración eficiente y responsable en la sociedad. Esta formación implicaría la capacidad y competencia de sostener diálogos críticos orientados a la búsqueda de la verdad teórica y práctica, en los que se exponen y examinan razones y puntos de vista diversos y divergentes, y de soluciones pacíficas y justas para los problemas y conflictos que surjan en el marco de una sociedad democrática.

La formación de especialistas y expertos tendría que incluir una capacitación que conduzca al desarrollo de competencias discursivas, éticas y culturales. En este sentido, los especialistas y expertos universitarios son -deberían ser- personas capacitadas no sólo para aumentar metódica y sistemáticamente el conocimiento sino también para colaborar competentemente en la búsqueda de sentido y en la orientación de acciones y decisiones que tiendan a lograr una articulación responsable entre los principios éticos universales y la realidad histórica (en sus contextos sociales y culturales). Por ello, los especialistas y expertos tienen que ser "personas de principios" y, a la vez, personas conocedoras de su propia cultura y del contexto histórico de aplicación.

\subsection{Universidad y sociedad civil.}

Lo que vincula intrínsecamente a la Universidad con la sociedad civil no es nada más (ni nada menos) que una búsqueda: la búsqueda conjunta, pública y sin restricciones de la verdad, tanto teórica como práctica. El discurso público, con sus exigencias de diálogo crítico y participativo, tiene una gran relevancia teórica, práctica y metodológica para el examen crítico de todas aquellas cuestiones que hacen a una convivencia libre, pacífica y justa, y ofrece un espacio privilegiado de convivencia en el que, por principio, no pueden tener cabida el dogmatismo y los privilegios irrazonables.

En principio, quizá nadie -o muy pocos- pongan en duda que la solución dialógica de los problemas y conflictos a nivel no sólo del mundo de la vida, sino también institucional y global, es un desideratum o un ideal a alcanzar. Sin embargo, a causa de las diversas ideologías y divergentes teorías que compiten en el mundo contemporáneo, y en vista de reiterados fracasos por alcanzar acuerdos perdurables sobre la base exclusiva del diálogo, a muchos les podría parecer ingenuo u "utópico" pensar en la eficacia del diálogo. Pensemos, por ejemplo, en que los diálogos mantenidos en la ONU no han podido detener la guerra contra Irak, y una vez más una concepción particular de la paz y la libertad se impuso, no por medio del discurso argumentativo, sino por la violencia y a espaldas de la comunidad internacional.

Todas estas observaciones y estos cuestionamientos son dignos de ser pensados. Es verdad que el acuerdo con los otros no es cosa sencilla, y que el diálogo aparece a menudo, sobre todo a la hora de solucionar problemas y zanjar conflictos, como una alternativa débil, quizá demasiado débil frente a las posibilidades que ofrecen la persuasión, la retórica, el engaño o el poder. Sin embargo, no es menos cierto que el

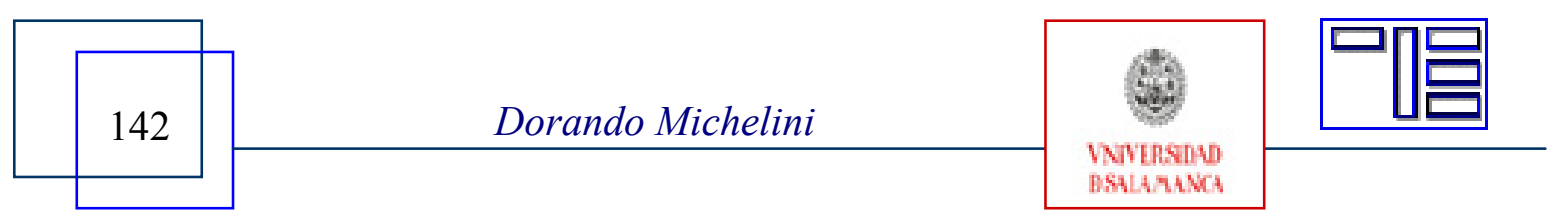


diálogo es la única posibilidad que tenemos de alcanzar un consenso con los otros, esto es: un entendimiento que no se restrinja al ámbito de las relaciones estratégicas y que, por ende, respete las normas éticas básicas de autonomía y libertad. ${ }^{3}$ Es por ello que es difícil vislumbrar una alternativa éticamente relevante que pueda sustituir al discurso argumentativo como lugar privilegiado del espacio público en general $-\mathrm{y}$ de la Universidad, en particular- para la resolución libre, justa y pacífica de los problemas y conflictos, esto es: para convivir en paz, a pesar de las diversas comprensiones de la verdad y de la dificultad de resolver, de modo convincente y general, los problemas y conflictos.

La intelección de que nadie tiene nada -o muy poco- asegurado en el horizonte concreto de esta búsqueda conjunta de la verdad, y de que las certezas absolutas en el ámbito práctico y las garantías de éxito en lo que respecta a la articulación histórica e implementación fáctica de una alternativa cultural humanamente válida son escasas, tendría que llevarnos a comprender que la única posibilidad de interactuar responsablemente en el ámbito institucional dentro de la Universidad, y, en el ámbito social, en la interacción entre Universidad y sociedad civil, en el sentido de contribuir a la configuración de una convivencia libre, pacífica y justa, es la cooperación dialógica en un discurso público solidario, abierto e irrestricto, con todos los implicados y afectados, en vistas de una autorrealización humana integral.

\section{4.- UNIVERSIDAD, DEMOCRACIA Y DISCURSO.}

En consonancia con lo expresado hasta aquí, puede sostenerse que en una sociedad democrática, la deliberación pública, abierta e irrestricta se presenta no sólo como un derecho de los ciudadanos sino, incluso, como el lugar privilegiado que tiene la sociedad civil para la toma de decisiones intersubjetivamente vinculantes. El discurso público puede ser comprendido como un procedimiento privilegiado que posibilita un tratamiento racional e imparcial de los problemas y abre un horizonte de solución pacífica y razonable de los conflictos que tienen lugar en el mundo de la vida a causa de las distintas pretensiones de verdad teórica y práctica.

Más aún, cuando real o aparentemente ninguna solución razonada aparece como aceptable, el mantenimiento del discurso público posibilita sostener la convivencia en el disenso: el discurso público se presenta como un mecanismo que frena la violencia y, mediante el diálogo, la confrontación en vista de las aspiraciones teóricas y prácticas divergentes puede ser contenida dentro de los límites del respeto mutuo.

La Universidad constituye un espacio público adecuado no sólo para la búsqueda del conocimiento científico-tecnológico metódica y sistemáticamente orientado, sino también para la reflexión racional y la búsqueda no dogmática de la verdad, y, con ello, para la formación discursiva de una conciencia ética y crítica, basada en la libertad y la corresponsabilidad solidaria. La Universidad conforma así un espacio de responsabilidad institucional frente a la sociedad para la formación de personas capaces

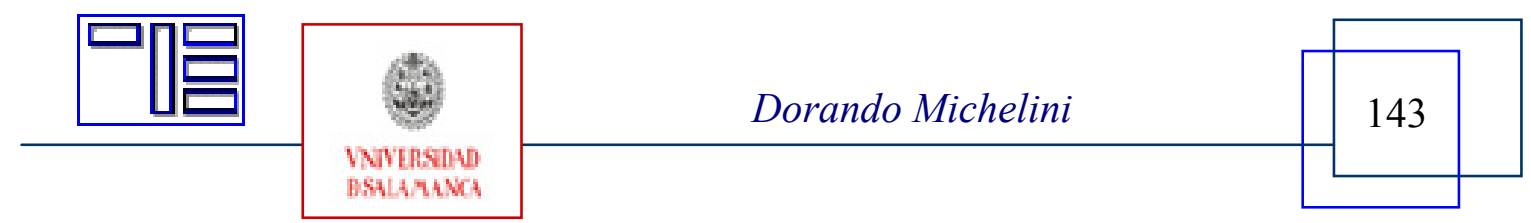


Revista Electrónica Teoría de la Educación.

Educación y Cultura en la Sociedad de la Información.

http://www.usal.es/teoriaeducacion

Vol. 9. No 2. Mayo 2008

de analizar y evaluar la realidad social, y para la deliberación racional y razonable de todas aquellas cuestiones que implican a todos y a cada uno de los ciudadanos de una comunidad. En este sentido, la Universidad configura un lugar privilegiado de la sociedad civil en el que los ciudadanos buscan nuevos conocimientos en las diferentes especialidades, pero donde también se preparan para participar responsablemente en la tematización, discusión y resolución de todas las cuestiones teóricas y prácticas socialmente relevantes.

La configuración de una auténtica sociedad democrática está sustentada así en la idea de una sociedad civil en la que el discurso público busca institucionalizarse en todos los niveles como un procedimiento ético, racional y razonable, para el tratamiento y la resolución de los problemas públicos relevantes que conciernen a todos los ciudadanos.

El discurso argumentativo posee una relevancia no sólo teórica y ética, sino también política. La democracia se sustenta y legitima en la idea de un discurso argumentativo como procedimiento para el examen y la discusión racional y razonable de todos aquellos asuntos que tienen pretensión de validez y vincularidad intersubjetiva en una sociedad. ${ }^{4}$ En el sistema democrático, la formación política de la voluntad se conecta "directamente con el principio de discusión general y libre de dominación" (Habermas, 1986, 113). En el contexto de sociedades democráticas, abiertas y pluralistas, esto significa aproximadamente lo siguiente: las normas que pretendan validez $\mathrm{y}$ vincularidad general (al igual que las pretensiones de verdad en el ámbito teórico, y que las pretensiones de corrección en el ámbito práctico) tienen que ser expuestas, analizadas y resueltas en discursos prácticos, en los cuales y a través de los cuales las leyes y normas de las instituciones básicas de la sociedad tienen que poder hallar la aprobación de todos los afectados, siempre que todos y cada uno de ellos puedan participar sin restricciones "en los discursos de formación de la voluntad en cuanto sujetos libres e iguales" (Habermas, 1976: 279) ${ }^{5}$.

Apel y Habermas coinciden en señalar que el principio del discurso orientado al consenso es el único criterio racional y metodológicamente relevante tanto para la solución racional de las cuestiones relacionadas con las pretensiones de verdad teóricas y prácticas, como para los asuntos atinentes a la legitimación de las normas e instituciones políticas en un sistema democrático. ${ }^{6}$ Por lo tanto, sólo podemos hablar de democracia en sentido auténtico cuando la formación de la voluntad y la toma de decisiones están articuladas con el discurso público, abierto e irrestricto ${ }^{7}$.

La democracia, más allá de todas las críticas a sus debilidades y falencias, es uno de los sistemas históricos de convivencia que permite aportar no sólo ejemplos concretos de convivencia, sino también fundamentar teóricamente los alcances y límites de una articulación entre libertad y conflictividad.

La sociedad democrática ofrece las condiciones adecuadas de libertad y pluralismo para el desarrollo del pensamiento científico, para la búsqueda de la verdad y el surgimiento

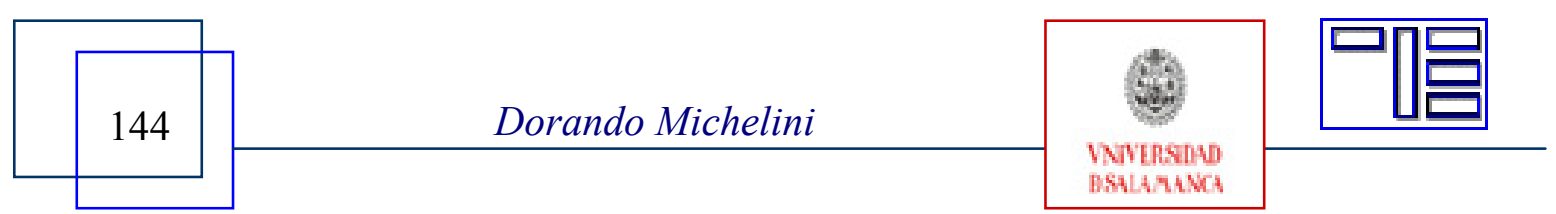


de un pensamiento crítico, y, con ello, para la configuración de una Universidad abierta al debate, en el que es posible presentar y examinar propuestas diversas y divergentes. Una Universidad que se sustenta en el diálogo amplio, crítico y sin exclusiones permite la disputa pública de diversas pretensiones de verdad teórica y práctica, y, a su vez, sienta las bases de valores fundamentales para una convivencia democrática.

De acuerdo con ello, tanto un sistema político democrático como la Universidad podrán autocomprenderse plenamente como tales en la medida en que el criterio de legitimación que predomine en sus respectivas estructuras y en su funcionamiento responda a los criterios generales de racionalidad esbozados, esto es: en la medida en que hagan depender su institucionalidad de procesos no excluyentes de participación y del consenso discursivo de todos los afectados. En definitiva, el grado de emancipación y humanidad de una sociedad democrática y de una Universidad crítica se corresponde con la capacidad que ellas tengan para asegurar, sin restricciones ni exclusiones, discursos libres y públicos de entendimiento e interacción.

\section{5.- BIBLIOGRAFÍA.}

ADORNO, Th. y M. Horkheimer (1970). Dialéctica del Iluminismo. Madrid: Editorial Totta.

ADORNO, Th. W. (1987). Der Positivismusstreit in der deutschen Soziologie (12 ${ }^{\mathrm{a}}$. Edición). Darmstadt: Editorial Luchterhand.

APEL, K.-O. (1973). Transformation der Philosophie (2 T.). Frankfurt am Main: Editorial Suhrkamp.

APEL, K.-O. (1978). "Der Ansatz von Apel”. En Oelmüller (ed.) (1978), Transzendentalphilosophische Normenbegründungen, Paderborn: Editorial Schöningh.

APEL, K.-O. (1987). Die philosophische Idee der Universität. Versuch einer kritischen Rekonstruktion und zeitgemässen Applikation, (manuscrito).

APEL, K.-O. (1988): Diskurs und Verantwortung. Das Problem des Übergangs zur postkonventionellen Moral. Frankfurt am Main, Editorial Suhrkamp.

BÖHME, G. (1992). Natürlich Natur (Über Natur im Zeitalter ihrer technischen Reproduzierbarkeit). Frankfurt am Main: Editorial Suhrkamp.

BOURDIEU, P. (1999). Contrafuegos. Reflexiones para servir a la resistencia contra la invasión neoliberal. Barcelona: Editorial Anagrama.

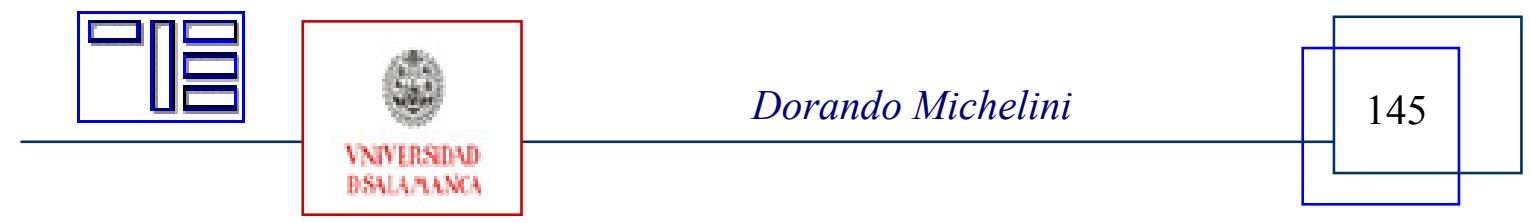


Revista Electrónica Teoría de la Educación.

Educación y Cultura en la Sociedad de la Información.

http://www.usal.es/teoriaeducacion

Vol. 9. No 2. Mayo 2008

FORNET BETANCOURT, R. (Ed.) (2003). Culturas y poder. Interacción y asimetría entre las culturas en el contexto de la globalización. Bilbao: Editorial Desclée de Brouwer.

HABERMAS, J. (1971). Theorie und Praxis. Frankfurt am Main: Editorial Suhrkamp.

HABERMAS, J. (1976). Zur Rekonstruktion des Historischen Materialismus. Frankfurt am Main: Editorial Suhrkamp.

HABERMAS, J. (1981). Theorie des kommunikativen Handelns, T. 1. Frankfurt am Main: Editorial Suhrkamp.

HABERMAS, J. (1984). "Über Moralität und Sittlichkeit - Was macht eine Lebensform 'rational'?". En: H. Schnädelbach (1984): Rationalität, Frankfurt am Main Editorial Suhrkamp.

HABERMAS, J. (1984). Vorstudien und Ergänzungen zur Theorie des kommunikativen Handelns. Frankfurt am Main: Editorial Suhrkamp.

HABERMAS, J. (1985): Conciencia moral y acción comunicativa. Barcelona, Editorial Península.

HABERMAS, J. (1986). "Entgegnung", en: A. Honneth y Hans Joas (eds.), Kommunikatives Handeln. Beiträge zu Jürgen Habermas' "Theorie des kommunikativen Handelns". Frankfurt am Main: Editorial Suhrkamp.

HABERMAS, J. (1986). Ciencia y técnica como ideología. Madrid: Editorial Tecnos.

HEIDEGGER, M. (1984). La pregunta por la técnica. Santiago de Chile: Editorial Universitaria.

HORKHEIMER, M. (1969). Crítica de la razón instrumental. Buenos Aires: Editorial Sur.

IRRGANG, B. (2002). Technischer Fortschritt. Legitimitätsprobleme innovativer Technik. Paderborn: Editorial Schöningh.

JONAS, H. (1985). Technik, Medizin und Ethik. Zur Praxis des Prinzips Verantwortung. Frankfurt am Main: Editorial Insel.

JONAS, H. (1995). El principio de responsabilidad. Ensayo de una ética para la civilización tecnológica. Barcelona: Editorial Herder.

LYOTARD, J.-F. (1989). La condición posmoderna. Buenos Aires: REI.

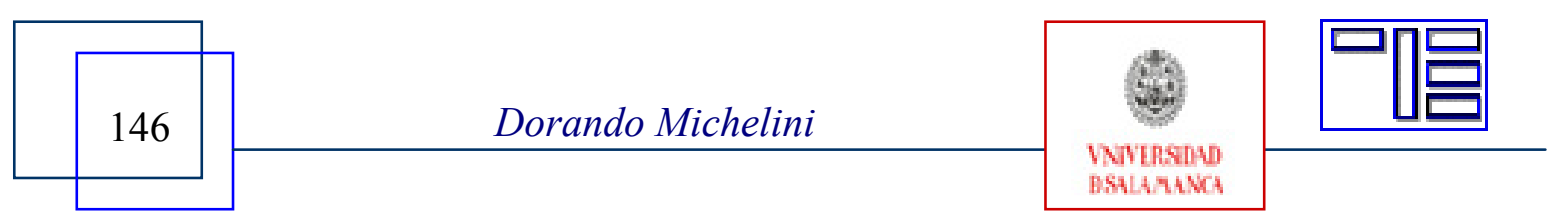


MICHELINI, D. J. (1998). La razón en juego. Río Cuarto: Editorial Universidad Nacional de Río Cuarto.

MICHELINI, D. J. (2000). Globalización, interculturalidad, exclusión. Estudios éticopolíticos. Río Cuarto: Ediciones del ICALA.

NOSSAL, G. J. V. (1988). Los límites de la manipulación genética. Barcelona: Editorial Gedisa.

OTEGA Y GASSET, J. (1961). "Meditación de la técnica", en Obras completas, Madrid: Editorial Revista de Occidente.

ROIG, A. A. (1998). La universidad hacia la democracia. Bases doctrinarias e históricas para la constitución de una pedagogía participativa. Mendoza: Editorial EDIUNC.

RORTY, R. (1983). La filosofia y el espejo de la naturaleza. Madird, Editorial Cátedra.

SALAS ASTRAIN, R. (2003): Ética Intercultural. (Re) Lecturas del pensamiento latinoamericano. Santiago de Chile: Editorial de la Universidad Católica Silva Henríquez.

SAMAJA, J. (2003). "Sobre la ciencia, la técnica y la sociedad. Para pensar la nueva agenda de la educación superior". En Ciencia, docencia y tecnología. $\mathrm{N}^{\mathrm{o}} 27$, Año XIV, disp. en http//www.revistacdyt.uner.edu.ar (15-11-2007).

\section{Notas:}

\footnotetext{
${ }^{1}$ La bibliografía sobre esta problemática es muy amplia. Aquí baste con mencionar las obras de Heidegger (1984), Ortega y Gasset (1961), Adorno (1970), Horkheimer (1969) y Habermas (1985).

${ }^{2}$ Así sostiene Samaja (2003), por ejemplo, que "lo cierto e indudable es que hoy la principal amenaza a la educación superior ha sido planteada por la desmesurada presión de las políticas mercantilistas que exaltan las formas societales lideradas actualmente por las gigantes empresas multinacionales, en detrimento de los estados nacionales, de sus diversas comunidades y, finalmente, de individuos que las integran, quienes corren el inminente peligro de ser privados de su condición de personas para quedar reducidos a una extensión unidimensional: productor-innovado-consumidor de las sociedades civiles, concebidas como agentes del mercado."
}

${ }^{3}$ En relación con la resolución discursiva de las pretensiones de verdad o de corrección, alguien podría cuestionar ciertamente que el resultado de un consenso válido, obtenido a través de un discurso argumentativo en las condiciones anteriormente mencionadas, podría no coincidir con la verdad. Esta apreciación tiende a cuestionar que la verdad tenga que ver necesariamente con el consenso. Esta observación respecto de que el acuerdo que se alcance en cada caso no necesariamente concuerda con la verdad es correcta en la medida que se piensa en consensos fácticos; el error de este cuestionamiento reside, sin embargo, en la imposibilidad de que alguien pueda ubicarse "más allá" del lenguaje y de la comunidad de comunica-

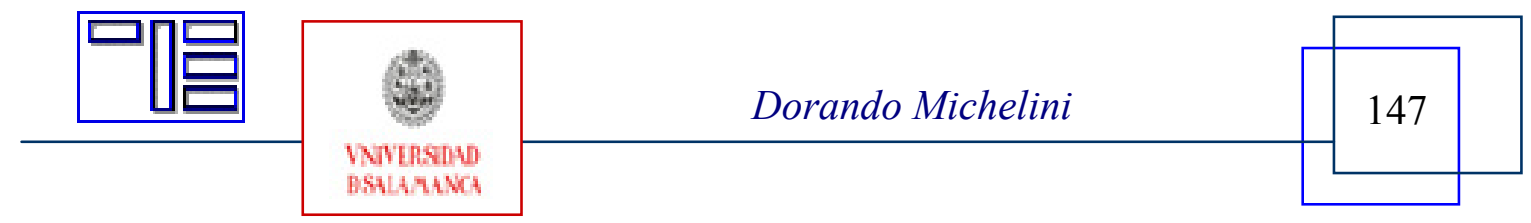


Revista Electrónica Teoría de la Educación.

Educación y Cultura en la Sociedad de la Información.

http://www.usal.es/teoriaeducacion

Vol. 9. No 2. Mayo 2008

ción para determinar en sentido absoluto la verdad de un asunto: las justificaciones consensuales remiten sólo a la validez racional de las respectivas pretensiones. Es por ello que el planteo ético-discursivo de la teoría de la verdad como consenso habla de "pretensiones" de verdad y de "condiciones de posibilidad de un conocimiento válido" en el marco de una comunidad ilimitada de comunicación. En consecuencia, cualquier corrección o cuestionamiento que se realice a un consenso alcanzado fácticamente tendrá que efectuarse mediante el lenguaje y dentro del marco de la comunidad de comunicación, esto es: mediante una nueva justificación de las pretensiones de validez.

${ }^{4}$ De acuerdo con la teoría habermasiana, la acción comunicativa funciona sin dificultades en el mundo de la vida mientras no aparezcan problemas teóricos o prácticos de entendimiento más o menos serios con los demás. Sin embargo, como todos sabemos, el mundo de la vida está minado de problemas, incertezas, desencuentros, asimetrías y exclusiones, por lo que el disenso y el conflicto aparecen a menudo como su materia prima. Y es justamente en el momento en que se produce la falta de entendimiento cuando hay que pasar de la interacción comunicativa al discurso. El discurso argumentativo aparece como la única forma racional de resolver conflictos de entendimiento con los otros (surgidos a causa de los cuestionamientos de las pretensiones teóricas y prácticas de validez) sin hacer uso de la violencia, sino apelando sólo a razones válidas.

${ }^{5}$ Originariamente, Habermas defendió la idea de que el discurso representa el "preanuncio de una forma de vida a realizarse en el futuro"; posteriormente se retracta de tal afirmación (Habermas, 1981, T. I, 110 ss.) y reconoce que las formas de vida, en cuanto "totalidades", no pueden ser sometidas a un enjuiciamiento moral (Habermas, 1984: 233).

${ }^{6}$ A diferencia de Habermas, para quien la democracia representa fundamentalmente un "procedimiento de entendimiento racional" (Habermas, 1971: 281), Apel sostiene que la democracia implica, además del procedimiento, "la realización de una idea" (Apel, 1978: 187) y "el intento de realizar la comunidad ilimitada de comunicación en el medio de la política" (Apel, 1973, T. II: 154). Con ello pretendo, además de marcar las diferencias entre ambos autores, destacar la idea de que el discurso público en el ámbito político, en cuanto procedimiento que implica la participación y el asentimiento de todos en aquellas cuestiones que posean un alcance general, es una determinada forma de legitimar intersubjetivamente las decisiones vinculatorias y tiene como finalidad no la erradicación de la coacción o del poder, sino la erradicación de la dominación a través de la "racionalización del poder político" qua autoridad que emana del consentimiento de todos los afectados.

${ }^{7}$ Claro está que no se trata de cualquier discurso, sino del discurso argumentativo, que tiene una estructura peculiar y características que pueden ser precisadas idealmente por el discurso filosófico. Se trata de un discurso que exige no sólo la igualdad de condiciones de todos los participantes al intervenir en los debates, sino también autonomía, distribución simétrica de chances y renuncia a la violencia para resolver los problemas comunes y los eventuales conflictos. Obviamente, estas características son difíciles de encontrar en los discursos prácticos políticos cotidianos, los cuales están transidos por las coacciones del tiempo y de los intereses, del poder y de la asimetría, de la retórica y la exclusión, de la pobreza y la miseria, etc. Por ello, la ética del discurso distingue entre la legitimación de la idea de la democracia y la aplicación o implementación de esta idea en las organizaciones e instituciones económicas, jurídicas y políticas.

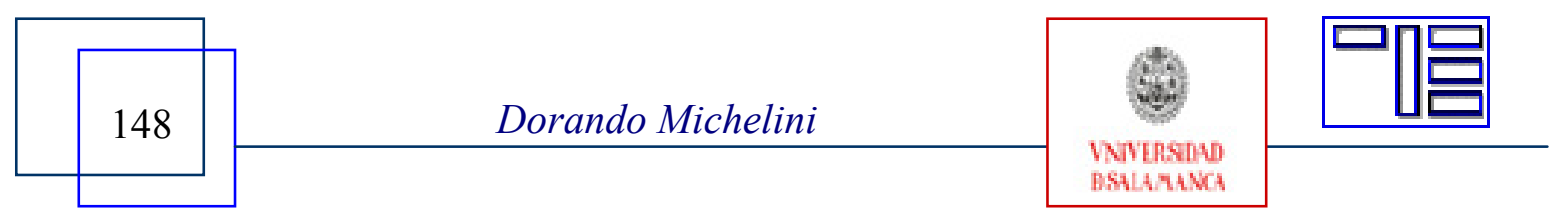


Para citar este artículo puede utilizar la siguiente referencia:

MICHELINI, Dorando (2008). Universidad, interculturalidad y discurso público. En APARICIO, Pablo (Coord.) Desde la diversidad hacia la desigualdad: ¿destino inexorable de la globalización? [monográfico en línea]. Revista Electrónica Teoría de la Educación: Educación y Cultura en la Sociedad de la Información. Vol. 9, nº 2. Universidad de Salamanca. [Fecha de consulta: dd/mm/aaaa].

$<\mathrm{http}: / /$ www.usal.es/ teoriaeducacion/rev_numero_09_02/n9_02_michelini.pdf $>$ ISSN 1138-9737

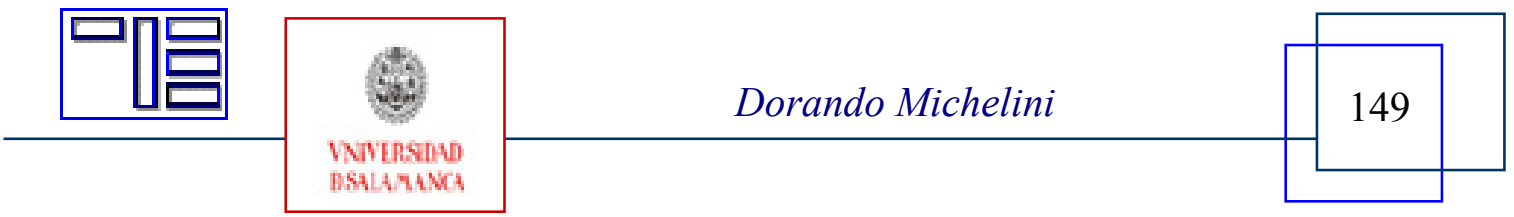

